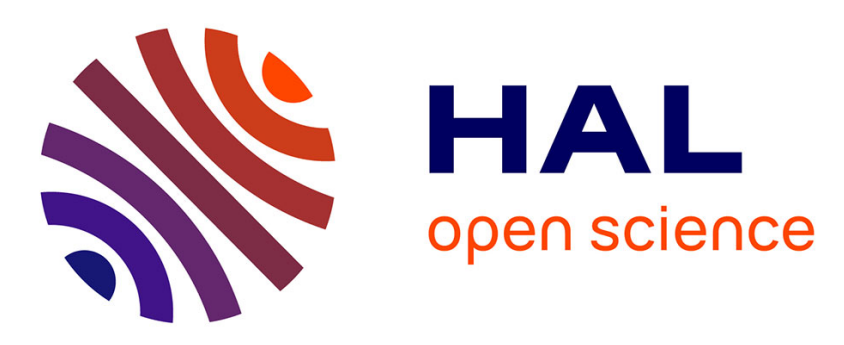

\title{
Modeling of the quasibrittle fracture of concrete at meso-scale: Effect of classes of aggregates on global and local behavior
}

Alexandre Gangnant, Jacqueline Saliba, Christian La Borderie, Stéphane

Morel

\section{To cite this version:}

Alexandre Gangnant, Jacqueline Saliba, Christian La Borderie, Stéphane Morel. Modeling of the quasibrittle fracture of concrete at meso-scale: Effect of classes of aggregates on global and local behavior. Cement and Concrete Research, 2016, 89, pp.35-44. 10.1016/j.cemconres.2016.07.010 . hal-02367593

\section{HAL Id: hal-02367593 \\ https://hal.science/hal-02367593}

Submitted on 21 Nov 2019

HAL is a multi-disciplinary open access archive for the deposit and dissemination of scientific research documents, whether they are published or not. The documents may come from teaching and research institutions in France or abroad, or from public or private research centers.
L'archive ouverte pluridisciplinaire HAL, est destinée au dépôt et à la diffusion de documents scientifiques de niveau recherche, publiés ou non, émanant des établissements d'enseignement et de recherche français ou étrangers, des laboratoires publics ou privés. 


\title{
Modeling of the quasibrittle fracture of concrete at meso-scale: Effect of classes of aggregates on global and local behavior
}

\author{
Alexandre Gangnant ${ }^{\mathrm{a}, *}$, Jacqueline Saliba ${ }^{\mathrm{a}}$, Christian La Borderie ${ }^{\mathrm{b}}$, Stéphane Morel ${ }^{\mathrm{a}}$ \\ a Université de Bordeaux, I2M UMR 5295, Institut de Mécanique et d'Ingénierie, Dept. Génie Civil et Environnemental (GCE), Bordeaux F-33000, France \\ ${ }^{b}$ Université de Pau et des Pays de l'Adour, SIAME EA 4581, Laboratoire des sciences de l'ingénieur appliquées à la mécanique et au génie electrique, Allée du Parc Montaury, \\ Anglet 64600, France
}

The computational power allows nowadays the development of mesoscopic models of concrete, based on finite element or lattices approaches, which represent the contribution of inclusions to the behavior of concrete. However, the smallest heterogeneities are often removed to these simulations for decreasing the computation time. In this paper, the effect of aggregate classes on the fracture behavior of a plain concrete is studied. Different simulations are performed from a mesoscopic model based on a diffuse meshing technique and Fichant's damage model, in which the smallest aggregates are successively removed from the granular skeleton to the benefit of a homogenized continuous mortar. The effects of these simplifications are then evaluated by comparing the fracture behaviors obtained to the one of the reference concrete. The results show the relevance of modeling all classes of aggregates in order to obtain an accurate description of the failure behavior of concrete.

\section{Introduction}

The accurate description of concrete behavior through a macroscopic constitutive law is complicated by the high heterogeneities of cementitious materials. During the fracture process, after elastic and homogeneous strains, a micro-cracked zone called the Fracture Process Zone (FPZ) appears before the peak load. This damaged zone, formed by matrix microcracking, debonding of cement-aggregate interface, and grain bridging, tends to develop and to localize into a macro-crack, and finally critically propagates during the loading. This nonlinear zone is responsible for the dissipation of the elastic energy stored in the structure due to stress transfer. Commonly such behavior is called quasi-brittle. The characterization of the FPZ (by numerical [1] or experimental [2] analysis) constitutes a major challenge for the understanding of concrete mechanical behavior. Concerning the global mechanical response, a softening behavior occurs due to strain/damage localization (LEFM cannot correctly represent the stress field), and this has to be explicitly taken into account [3]. Concrete is a composite material with significant heterogeneities which have an important influence on concrete behavior at failure. The structure of concrete can be considered as

\footnotetext{
* Corresponding author.

E-mail address: a.gangnant@i2m.u-bordeaux1.fr (A. Gangnant).
}

a multi-level hierarchy system (macro-meso-micro-nanolevel) [4]. In this case, interaction between various components of concrete is required in order to accurately simulate the softening behavior, the damage fields and the crack paths, and to investigate the influence of concrete composition on the macroscopic properties [5]. Currently the computational power of computers allows the development of many non-linear models at mesoscale which present a real interest for describing the complex failure of concrete, ranging from diffuse failure to localization and final discrete failure. Nevertheless, most mesoscopic models are forced to simplify the concrete microstructure, representing only the largest aggregates because of the difficulty of meshing the smallest aggregates and the corresponding increase in computation time. The main question is what is the consequence of such simplification of concrete microstructure on the relevance of the simulated behavior. Indeed, mechanical and physical properties of concrete are dependent on the volume fraction and properties of the constituents [6]. The mesolevel approach is useful for analyzing the influence of aggregates on the failure behavior of concrete which is affected by the size, shape and grading of aggregates [7]. Moreover, during the failure process, the crack pattern (tortuosity) is governed by the position of the coarse aggregates. Generally aggregates are the cause of tougher, stiffer and more ductile behavior of concrete as the volume fraction of fine and coarse aggregates is increased without changing their grading [8]. The use of non-linear models at mesolevel can be performed by applying the 


\section{Nomenclature}

$\begin{array}{ll}B_{t} & \text { Parameter of the damage evolution law } \\ C_{i j k l} & \text { Elastic stiffness tensor components } \\ D & \text { internal variable for damage } \\ E & \text { elastic modulus } \\ f g_{i} & \text { volume fraction of aggregates per class } i \\ f_{t} & \text { tensile strength } \\ F v g & \text { global volume fraction of aggregates } \\ G_{f} & \text { fracture energy } \\ h & \text { element size } \\ I_{1} & \text { First principal invariant of the effective stress } \\ J_{2} & \text { Second principal invariant of the deviatoric part of } \\ & \text { the effective stress } \\ K e_{i j} & \text { elementary stiffness matrix } \\ L_{R E V} & \text { length of the Representative Elementary Volume } \\ \emptyset_{i} & \text { aggregate diameter of the class } i \\ \emptyset_{\max } & \text { maximum aggregate diameter } i \\ \emptyset_{\min } & \text { minimum aggregate diameter } i \\ W_{F} & \text { Work of the fracture } \\ X_{i} & \text { first coordinate of an aggregate } \\ Y_{i} & \text { second coordinate of an aggregate }\end{array}$

\section{Greek symbols}

$\begin{array}{ll}\varepsilon_{k l}^{e} & \text { Elastic Strain tensor components } \\ \varepsilon_{e q} & \text { Equivalent Strain } \\ \eta_{g} & \text { second coordinate of a Gauss point } g \\ \xi_{g} & \text { first coordinate of a Gauss point } g \\ \tilde{\sigma}_{i j} & \text { Effective Stress tensor components } \\ \sigma_{i j} & \text { Stress tensor components } \\ \omega_{g} & \text { Weight associated with the Gauss point } g\end{array}$

\section{Abbreviations}

$\begin{array}{ll}\text { BFGS } & \text { Broyden-Fletcher-Goldfarb-Shanno algorithm } \\ \text { FPZ } & \text { Fracture Process Zone } \\ \text { H } & \text { homogenized } \\ \text { HM } & \text { homogenized mortar } \\ \text { HPC } & \text { high performance concrete } \\ \text { ITZ } & \text { Interfacial Transition Zone } \\ \text { M } & \text { Missing } \\ \text { P } & \text { Present } \\ \text { REV } & \text { Representative Elementary Volume }\end{array}$

Finite Element Method [9], the Discrete Element Method [10] or the resource-intensive lattice method [11]. Because of the difficulty of meshing together small and large aggregates, most authors merely model the largest aggregates. The matrix is then composed with a mortar that accounts for the smallest aggregates.

The aim of this paper is to present the influence of small aggregates on the failure behavior of plain concrete by the use of a damage constitutive law simulated from a Finite Element Model established at mesolevel, i.e., taking into consideration the roles of the cement matrix and of the aggregates. The damage model used for the simulation of the fracture behavior of concrete is an isotropic damage model developed by Fichant et al. [12], that takes into account the unilateral effects, used at the mesoscale of concrete for both aggregates and matrix. The model is implemented in the finite elements code Cast $3 \mathrm{M}^{\odot}$. Moreover, a diffuse meshing method is used, whereby the matrix and aggregates properties are projected on the shape functions of the finite element mesh. For this study, the accurate representation of the aggregates compactness is priority, that is a reason why the Interfacial Transition Zone (ITZ) is not represented in this mesoscopic model. Moreover, its mechanical parameters are difficult to assess and the use of a diffuse meshing method may offset this issue. Thus, in this mesoscopic model, a "natural" ITZ takes place since elastic and fracture parameters contrasts lead to a stress concentration and, thus, damage localizes at the paste-aggregates interface. In the following the parameters used for cement paste and aggregates refer to normal concrete for which intragranular cracking is expected. Indeed, in a plain concrete the aggregates are not expected to damage. Thus, in this case, the aggregates can be seen as strong inclusions and lead to tortuous crack path and consequently to a crack-path dependent fracture energy. This concrete configuration seems to be more appropriate in order to study the effect of aggregates classes on the fracture behavior compared to the case of high performance concretes (HPC). The model is also able to capture trans-granular cracking by adopting appropriate parameters which is more appropriate for high performance concretes.

In this paper, the isotropic damage model [12] is presented along with the generation process of the mesostrucrure of concrete, as well as the estimation procedure of the mechanical and fracture properties of the aggregates and the mortar matrix. The computing strategy used to investigate the influence of aggregate classes on the fracture behavior is then explained. Finally, the relevance of modeling all or part of the aggregate classes of concrete by a numerical homogenization method is studied and discussed.

\section{Constitutive damage model}

\subsection{Principle of the constitutive model}

The damage model used is the isotropic damage model developed by Fichant [13], which is an extension of Mazars' model [14] taking into account the unilateral effect of concrete and inelastic strains (plasticity). The microcracking effect is directly linked to the internal state damage variable $D$. This damage variable is ranged from 0 , for an undamaged material, to 1 , for a fully damaged material. The notions of damaged and undamaged lead to the concept of effective stress $\tilde{\sigma}(1)$, which represents the necessary stress to apply to an undamaged material element so that it deforms the same way as a damaged element under total stress $\sigma(2)$.

$\tilde{\sigma}_{i j}=C_{i j k l}^{0}: \varepsilon_{k l}^{e}$,

where $\varepsilon_{k l}^{e}$ is the local elastic strain fields and $C_{i j k l}^{0}$ the initial isotropic elastic stiffness tensor. The total stress is described by:

$\sigma_{i j}=C_{i j k l}^{\text {damaged }}: \varepsilon_{k l}^{e}$,

where $C_{i j k l}^{\text {damaged }}$ is the damaged material stiffness tensor.

Based on Eqs. (1) and (2), the relation between total and effective stress can written as follows:

$\sigma_{i j}=C_{i j k l}^{\text {damaged }}:\left(C_{k l m n}^{0}\right)^{-1}: \tilde{\sigma}_{m n}=(1-D) \tilde{\sigma}_{m n}$,

where $\left(C_{k l m n}^{0}\right)^{-1}$ is the initial compliance tensor and $D$ the scalar variable of the isotropic model. The evolution of the isotropic damage variable $D$ is expressed as an exponential law:

$D=1-\frac{\varepsilon_{d 0}}{\varepsilon_{e q}} \exp \left(B_{t}\left(\varepsilon_{d 0}-\varepsilon_{e q}\right)\right)$

where $B_{t}=\frac{h f_{t}}{G_{f}-0.5 f_{t} \varepsilon_{d 0} h}$ is a damage parameter driving the shape of the strain softening and in which $f_{t}$ is the tensile strength, $G_{f}$ is the fracture energy and $h$ corresponds to the size of the finite element (in 
2D analysis $h$ corresponds to the square root of the element surface if the element is isotropic). Moreover, in Eq. (4) $\varepsilon_{d 0}$ is the damage threshold expressed in strain, i.e., $\left(\varepsilon_{d 0}=\frac{f_{t}}{E}\right)$ where $E$ is the Young modulus, and $\varepsilon_{e q}$ is the equivalent strain defined by Mazars which drives damage evolution and describes the state of local extension induced by stress:

$\varepsilon_{e q}=\sqrt{\Sigma_{i=1}^{3}<\varepsilon_{i}^{e}>_{+}^{2}}$,

where $\varepsilon_{i}^{e}$ is the principal elastic strain in the $i$-direction and \langle\rangle$_{+}$ corresponds to the Mac Cauley operator. The mesh dependency is controlled through the energetic regularization based on the Crack Band Theory [15] (i.e., the fracture energy $G_{f}$ related to the softening behavior). The fracture zone is then considered with a certain width $h$ where micro-cracks are uniformly distributed. The energy dissipation due to fracture per unit length is therefore constant with $G_{f}=h \int_{0}^{\infty} \sigma d \varepsilon$. By replacing $\sigma$ with the damage evolution law, the relation between the parameter $B_{t}$, the fracture energy and the element size can be obtained [16]. To conclude, the post-pic behavior is adjusted for each element size $h$ in order to dissipated the same amount of fracture energy. The damage constitutive law is applied on each Gauss point (which represents either the paste or an aggregate). After the estimation of an effective stress $\tilde{\sigma}$ calculated from the total strain increment (from an imposed displacement), the internal variable $D$ is calculated thanks to the Eq. (4). Then, the total and the inelastic stresses tensors are estimated from the isotropic damage variable $D$.

\subsection{Model parameters}

As already mentioned, the damage model takes place at mesoscale, i.e., the fracture behaviors of the aggregates and of the mortar matrix follow the isotropic damage model with damage parameters corresponding to each component. The input parameters (Young modulus, Poisson's ratio, tensile strength, and fracture energy) for cement matrix and aggregates are summarized in Table 1. The most of used properties are from literature [17,18]. Note that in our case, limestone aggregates were simulated and this lead to the use of a low Young's modulus of the aggregates [19]. As a plain concrete is studied, the damage threshold $\varepsilon_{d 0}$ of the aggregates might be higher than the cement paste,thus, the choice of a "low" but "realistic" Young's modulus has been made. Nonetheless, the elastic's modulus of aggregates does not strongly influence the crack path since this latter is mainly governed by fracture properties. Note that the elastic mismatch between matrix and aggregates creates stress concentrations at the aggregates' interfaces and promotes the development of damage in these areas.

\subsection{Solving procedure}

The finite element problem is solved using the classical procedure for non linear problems that is programmed within the Cast3M code. This procedure is based on an implicit time scheme and a quadratic scheme of kind BFGS [20] for the material non linearities. Small time step is adopted in order to avoid bifurcated solutions that would unless occur.

Table 1

Elastic and fracture parameters for the cement matrix and the aggregates.

\begin{tabular}{llll}
\hline & Properties & Aggregates & Cement paste \\
\hline Elastic properties & $E(\mathrm{GPa})$ & 35 & 25 \\
& $\nu$ & 0.2 & 0.2 \\
Fracture properties & $f_{t}(\mathrm{MPa})$ & 6 & 3 \\
& $G f\left(\mathrm{~J} / \mathrm{m}^{2}\right)$ & 60 & 20 \\
\hline
\end{tabular}

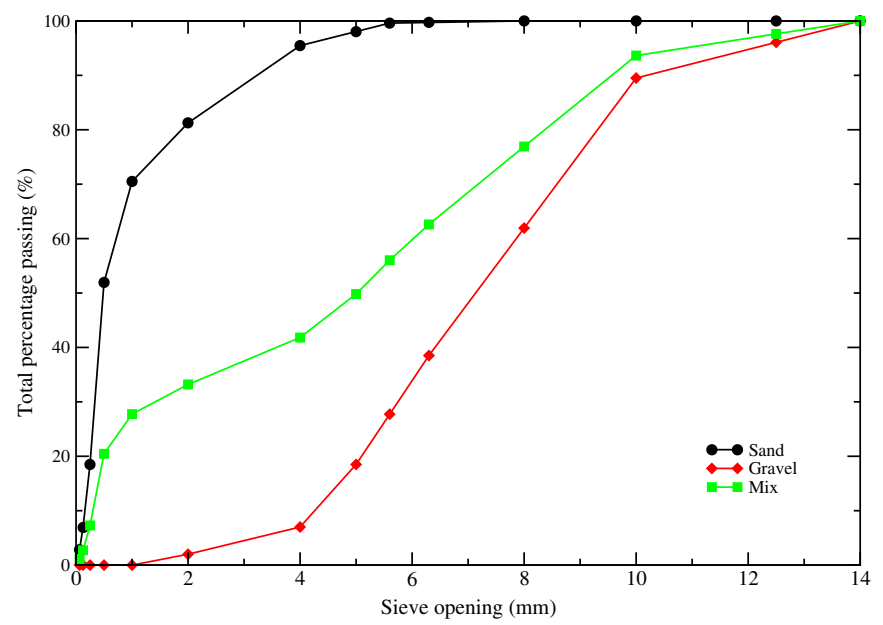

Fig. 1. Size distribution of aggregates corresponding to the M75C concrete.

\section{Mesostructure}

\subsection{Generation of the mesostructure}

The generation of the mesostructure consists of randomly placing the aggregate particles in the matrix on the basis of a stochasticheuristic algorithm: the aggregates are placed one by one from the largest to the smallest (take and place approach) [21]. If a dropped aggregate overlaps one already in place, a new draw is carried out until no overlapping remains. The spatial distribution of the aggregates follows the aggregate grading curve of the concrete studied (M75C concrete) given by Hager [22] (Fig. 1). Ten classes of aggregates are generated according to Fig. 1 for a global volume fraction of aggregate of $70 \%(F v g)$. The volume fraction of each class of aggregates $\left(f g_{i}\right)$ and their diameters $\left(\emptyset_{i}\right)$ used in the numerical concrete are presented in Table 2 .

For this study the "digital concrete" model is developed in two dimensions due to the large number of simulations, and hence aggregates are simply represented with a circular shape [23]. After each draw (following the previous algorithm), the position of all aggregates (coordinates $X_{i}, Y_{i}$ of the center of a particle with $\emptyset_{i}$ diameter) is known in the "mesoscopic box" and can be imported in the finite element code for the meshing process.

\subsection{Diffuse meshing method}

The mesoscopic mesh is obtained by using the diffuse meshing method [18] in which the heterogeneous material properties are directly projected on the Gauss points of any finite element mesh (regular or not). This method serves to represent different materials in one finite element; additionally, the shape of the aggregates is not really meshed. In our case the mesh is a regular grid made up of four-node quadrilateral elements (Fig. 2). The elementary stiffness

Table 2

Volume fraction of each class of aggregates

\begin{tabular}{llllll}
\hline $\mathrm{N}^{\circ}$ Class $_{i}$ & $\emptyset_{i}[\mathrm{~mm}]$ & $f g_{i}[\%]$ & $\mathrm{N}^{\circ}$ Class $_{i}$ & $\emptyset_{i}[\mathrm{~mm}]$ & $f g_{i}[\%]$ \\
\hline 1 & 1 & 27.750 & 6 & 6.3 & 6.576 \\
2 & 2 & 5.444 & 7 & 8 & 14.324 \\
3 & 4 & 8.616 & 8 & 10 & 16.713 \\
4 & 5 & 7.999 & 9 & 12.5 & 3.979 \\
5 & 5.6 & 6.211 & 10 & 14 & 2.388 \\
\hline
\end{tabular}


matrix $K e_{i j}$ is calculated as a function of the distribution of different materials (aggregates and matrix).

$K e_{i j}=\sum_{g=1}^{N} \omega_{g} B_{k i}\left(\xi_{g}, \eta_{g}\right) C_{k l}\left(\xi_{g}, \eta_{g}\right) B_{i j}\left(\xi_{g}, \eta_{g}\right)$

where $N$ is the number of Gauss points, $\omega_{g}$ is the weight associated with each Gauss point, $\xi_{g}$ and $\eta_{g}$ are the coordinates of Gauss Points, $B_{i j}$ is the gradient of the shape function matrix and $C_{k l}$ is the stiffness tensor of the material at Gauss point $g$. Unlike the exact meshing method, the diffuse meshing method allows an easy generation of the smallest aggregates. Nevertheless, the downside of using a regular grid is that the size of an element is totally dependent on the diameter of the finest aggregates. N'Guyen [18] showed numerically if the element size $h$ is less than the third of the minimum diameter $\left(\emptyset_{\min }\right)$, the fracture behavior obtained on the basis of the diffuse mesh technique is similar to the one obtained on the basis of an exact meshing method (see Section 2.2 of [18]). Moreover, Grondin et al. [24] showed that the stability of the results according to the random distribution of heterogeneities for an exact meshing method is obtained if the finite element size is equal to 0.8 times the smallest inclusion diameter.

An element size of $0.3 \mathrm{~mm}$ was chosen in our case:

$h<\frac{\emptyset_{\min }}{3} \simeq 0.33 \mathrm{~mm}$

This element size is used for all FE simulations (reference concrete, mortars, and simplified concretes described in the following). Thus, the regular FE mesh is the same for all configurations of concretes studied. The diffuse meshing method serves to represent a wide range of classes of aggregates according to the grading curve of concrete in strict compliance with the granular compactness.

In this study, the choice is made to simulate the experimental aggregate compactness as well as the smallest aggregates, to the detriment of the ITZ simulation which is not considered in the present model. Nevertheless, as shown below, the damage simulation from this model will occur at the matrix/aggregate "interface". Thanks to the stiffness mismatch between the two components, it is

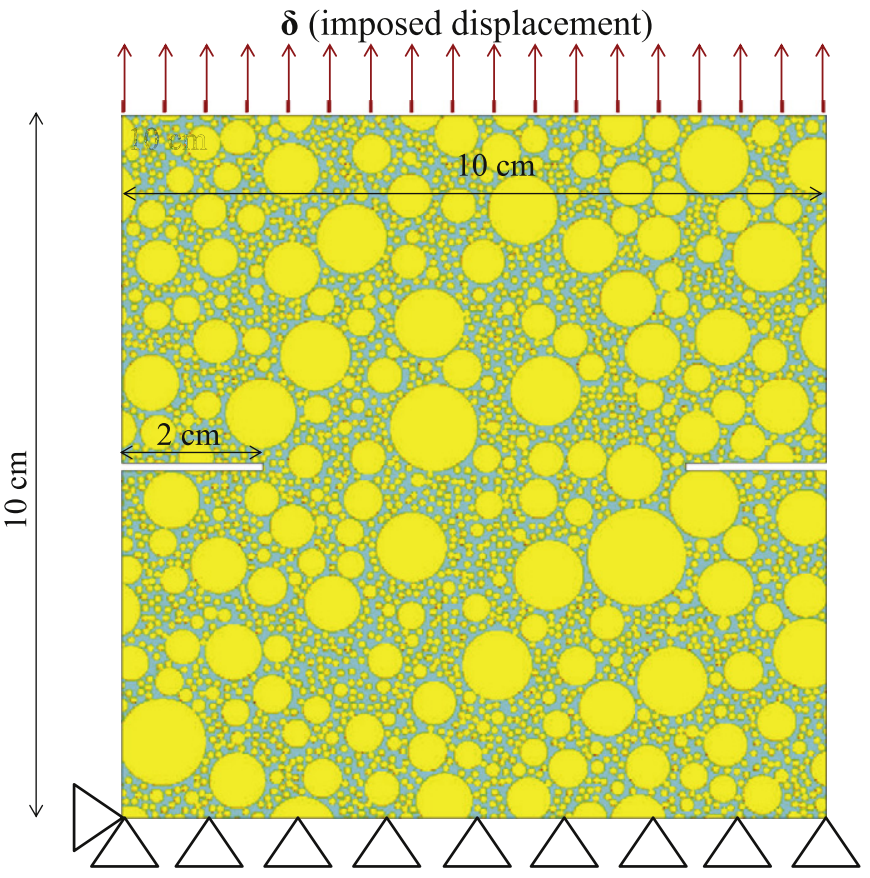

Fig. 3. Notched specimen loaded in tension (dimensions and boundary conditions)/ mesostructure corresponding to the reference one (all graduate classes).

in agreement with the expected experimental location of the damage in concrete. On the other hand, the simulation of all aggregate classes of concrete, and especially those corresponding to the smallest aggregates, involves extensive computation time due to necessarily fine meshes. It is therefore tempting to eliminate the smallest aggregates in order to enlarge the minimum size of the finite elements and hence to decrease the computation time. The main question concerns the influence of such a simplification of the microstructure on the global and local simulated fracture behavior of concrete. The answer to this question is the subject of the present study.

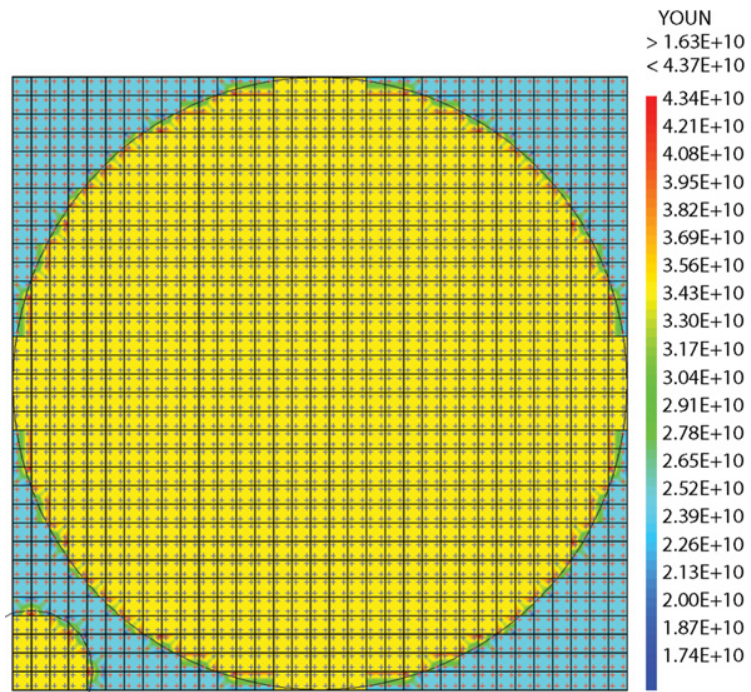

(a) view of one aggregate
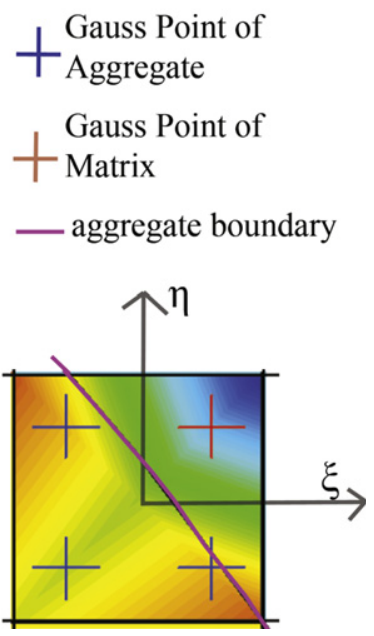

(b) close-up view of a finite element

Fig. 2. Local Young modulus values using the diffuse meshing method. (a) View of one aggregate (b) close-up view of a finite element. 


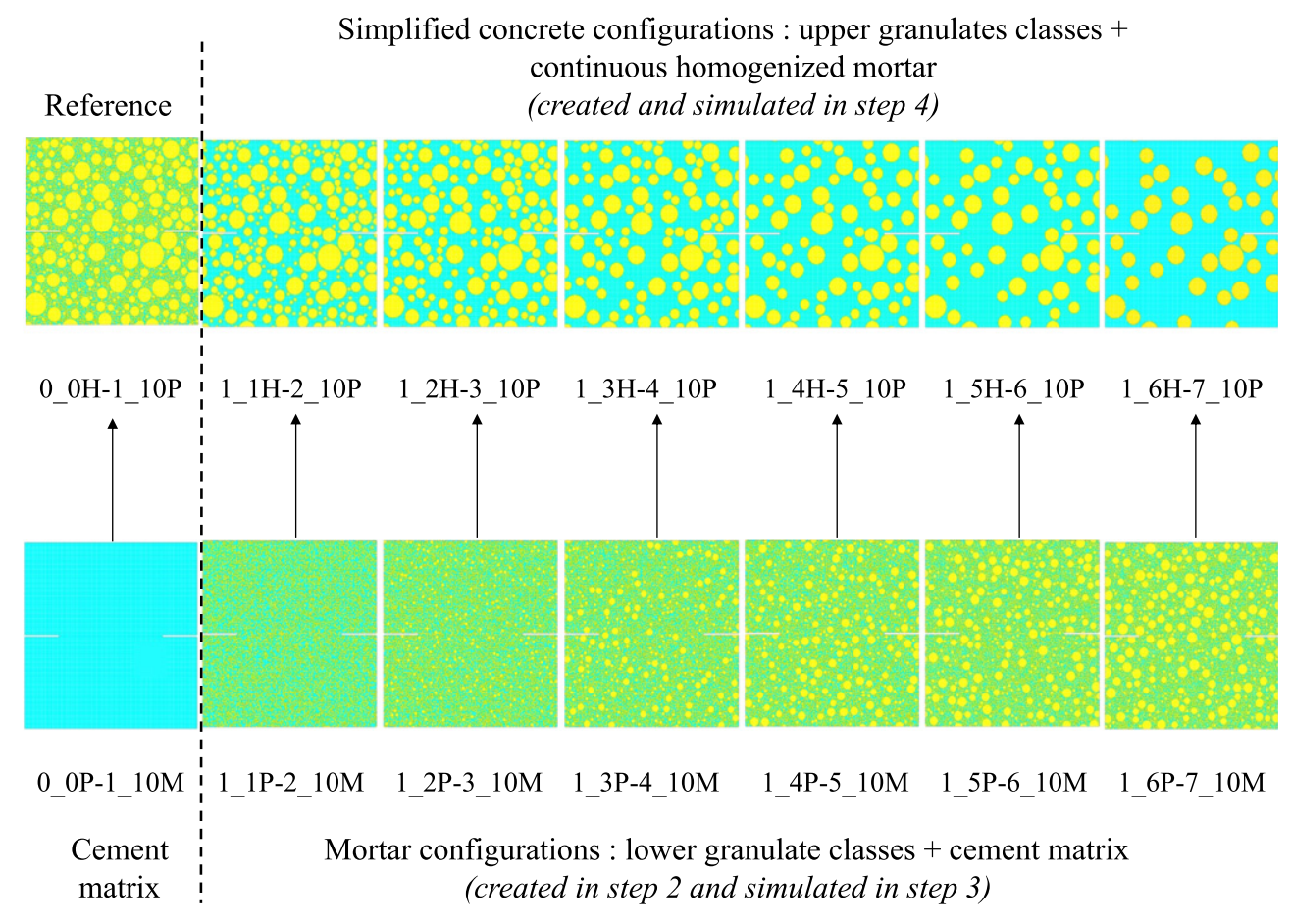

Fig. 4. Examples of the 6 different configurations of simplified concrete.

\section{Effect of the microstructure simplification on the simulated fracture behavior}

\subsection{Simulation samples}

The numerical simulations are conducted from a notched specimen loaded in tension under plane stress condition in 2D from an imposed displacement on the upper boundary of the specimen (as shown in Fig. 3). During the simulation, the displacement increment follows a geometric progression from $0.4 \mu \mathrm{m}$ micrometer to $300 \mu \mathrm{m}$. This kind of progression imposes low displacement increment around the peak load then, higher and higher displacement increment until the end of the simulation (corresponding to the typical asymptotic behavior of quasi-brittle materials). Nevertheless, the choice of the displacement increment is quite important for avoiding any converging problem during the resolution. The dimensions of the specimen are $10 \times 10 \mathrm{~cm}^{2}$ in order to have a Representative Elementary Volume (REV). According to Grondin [25], the length of $\operatorname{REV}\left(L_{R E V}\right)$ must be higher than 5 times the maximum diameter of aggregate $\left(\emptyset_{\max }=14 \mathrm{~mm}\right.$ so $\left.L_{R E V}>70 \mathrm{~mm}\right)$.

\subsection{Simulation procedure}

In order to answer the question of the effect of the microstructure simplification on the simulated fracture behavior, a comparison of the mechanical and damage responses between the reference configuration where all classes of aggregates are present and configurations corresponding to successive simplifications of the mesostructure of mortar are performed. The simplified configurations of concrete consist in successively removing the lower aggregate classes of the granular skeleton to the benefit of a mortar matrix; the mortar matrix thus considered is simulated as a continuous medium whose mechanical and fracture properties are estimated from a mortar constituted by the cement matrix and the lower aggregate classes. The simulation procedure comprises four successive steps:

1. Simulation of the numerical tensile test using the reference digital concrete where all the classes of aggregates $i=1$ to 10 are present (Table 2 ) and using the properties presented in Table 1.

2. Creation of mortar specimens comprising the cement matrix in which the lower aggregate classes are successively added. From a practical point of view, for a mortar specimen constituted by the cement paste and the 1 to $i$ aggregate classes, the mesostructure is simulated by replacing the upper classes

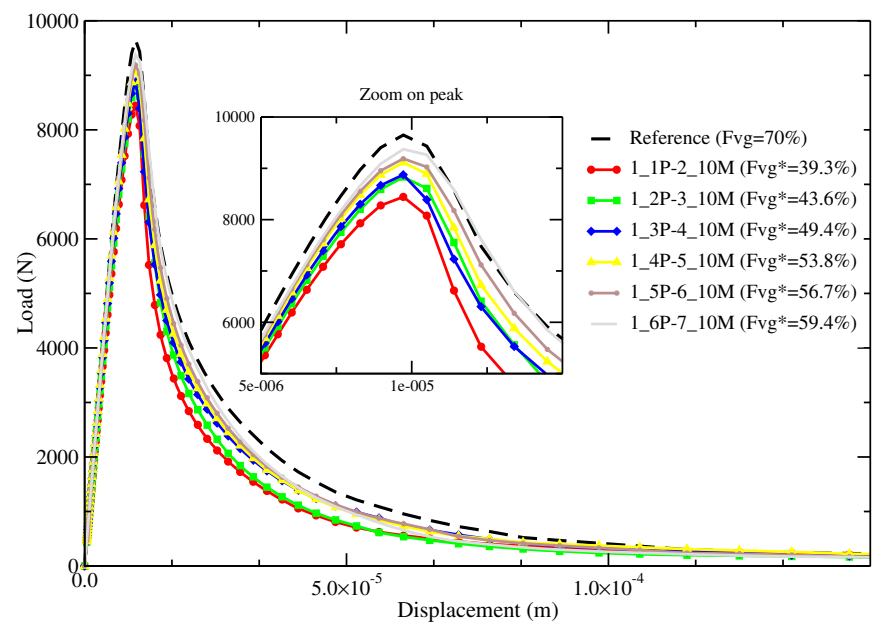

Fig. 5. Macroscopic response: average load-displacement curves for homogenized mortars. 
Table 3

Average properties of homogenized mortars.

\begin{tabular}{|c|c|c|c|c|c|c|c|}
\hline Configurations & Fvg* $^{*}$ & $\begin{array}{l}E_{\text {mean }} \\
(\mathrm{GPa})\end{array}$ & $\nu_{\text {mean }}$ & $\begin{array}{l}f_{\text {tmean }} \\
(\mathrm{MPa})\end{array}$ & $\begin{array}{l}G_{\text {fmean }} \\
\left(\mathrm{J} / \mathrm{m}^{2}\right)\end{array}$ & $\begin{array}{l}G_{f m i n} \\
\left(\mathrm{~J} / m^{2}\right)\end{array}$ & $\begin{array}{l}G_{f \max } \\
\left(\mathrm{J} / \mathrm{m}^{2}\right)\end{array}$ \\
\hline 1_1P-2_10 M & $39.3 \%$ & $28.25 \pm 2.45 e-3$ & $0.2 \pm 1.71 e-5$ & $2.81 \pm 0.039$ & $65.65 \pm 10.11$ & 45.43 & 85.87 \\
\hline 1_2P-3_10 M & $43.6 \%$ & $28.97 \pm 2.54 e-3$ & $0.2 \pm 1.90 e-5$ & $2.94 \pm 0.037$ & $65.08 \pm 9.60$ & 45.88 & 84.28 \\
\hline 1_3P-4_10 M & $49.4 \%$ & $29.52 \pm 3.86 e-3$ & $0.2 \pm 1.47 e-5$ & $2.95 \pm 0.030$ & $67.46 \pm 17.34$ & 50.61 & 84.31 \\
\hline 1_4P-5_10 M & $53.8 \%$ & $29.95 \pm 8.64 e-3$ & $0.2 \pm 2.29 e-5$ & $3.04 \pm 0.032$ & $73.69 \pm 15.45$ & 53.06 & 94.33 \\
\hline 1_5P-6_10 M & $56.7 \%$ & $30.24 \pm 1.36 e-2$ & $0.2 \pm 2.45 e-5$ & $3.05 \pm 0.058$ & $69.51 \pm 22.02$ & 47.90 & 91.12 \\
\hline 1_6P-7_10 M & $59.4 \%$ & $30.52 \pm 1.42 e-2$ & $0.2 \pm 2.27 e-5$ & $3.12 \pm 0.041$ & $70.79 \pm 19.05$ & 52.12 & 89.40 \\
\hline
\end{tabular}

(i.e., $i+1$ to 10 aggregate classes) by a mortar having the same cement paste/aggregates volume fraction ratio as in the reference concrete. Thus, the new global volume fraction $F v g_{1-i}^{*}$ of a mortar made up of the cement paste and the 1 to $i$ aggregate classes is obtained as:

$$
F v g_{1-i}^{*}=\frac{F v g \sum_{1}^{i} f g_{i}}{1-F v g \sum_{i+1}^{10} f g_{i}}
$$

The volume fraction per class of aggregate can be obtained by a proportional law using $F v g_{1-i}^{*}$. The mortar is identified by the following nomenclature: "1_(i)P-(i+1)_10M" where $i$ is the highest class of aggregates present in the mortar. $P$ means "Present" and $M$ means "Missing". In this study, classes of aggregates are deleted until $\emptyset=6.3 \mathrm{~mm}$ (class $n^{\circ} 6$ ). Note that only 6 different mortars are considered in this study, i.e., consisting in the successive inclusions of the 6 lower aggregate classes.

3. Estimation of the elastic and fracture properties of mortar “1_(i)P-(i+1)_10M" defined in step 3. Young modulus $\left(E_{H M}\right)$, Poisson coefficient $\left(\nu_{H M}\right)$ and tensile strength $\left(f_{t H M}\right)$ are estimated from the load-displacement response obtained from the simulation of a tensile test on an un-notched specimen (specimen shown in Fig. 3 but without notches), while the fracture energy $\left(G_{f H M}\right)$ is estimated from a notched specimen (Fig. 3) in order to obtain a single final crack and so a relevant value of the fracture energy. Note that the tensile strength $\left(f_{t H M}\right)$ is estimated from an un-notched specimen, thus avoiding the notch effect.

4. Finally, the last step consisted in simulating concrete with a simplified mesostructure, i.e., comprising the $i+1$ to 10 upper aggregate classes and a mortar (continuous medium) whose homogenized properties correspond to those estimated from step 3 “1_(i)P-(i+1)_10M" as shown in Fig. 4. The nomenclature of the simplified concrete is "1_(i)H-(i+1)_10P” where $H$ means "Homogenized" and $P$ means "Present". Note that the properties of the present aggregates in the simplified concrete remain equal to those defined in Table 1 . Six simplified mesostructures of mortar are simulated as shown in Fig. 4, and their load-displacement responses obtained from the tensile test on the notched specimen (Fig. 3) are compared to the reference one obtained in step 1 . On the other hand, in order to obtain an average fracture behavior for each concrete configuration, i.e., a fracture behavior independent of the aggregate location in concrete, ten draws of the mesostructure are performed for each concrete configuration (reference concrete configuration and simplified ones) as well as for each mortar shown in Fig. 4. Moreover, note that the 10 draws of each simplified concrete are obtained for the 10 draws of the reference concrete. Thus, the effect of the smallest aggregates on the damage location and on the crack path (tortuosity) can also be studied by comparing the corresponding reference and simplified configurations.

\subsection{Identification of homogenized mortar properties}

For each of the six homogenized mortars shown in Fig. 4, 10 draws are simulated for the un-notched specimen - estimation of $E_{H M}, \nu_{H M}, f_{t H M}$ - and 10 draws are simulated for the notched one estimation of $G_{f H M}$ - i.e., 120 simulations. Fig. 5 shows the average load-displacement responses (average of the 10 draws) obtained in the case of notched mortar specimens. The 120 load-displacement responses are analyzed and lead to the average and standard deviations of elastic and fracture properties reported in Table 3. Note that, according to the RILEM 50-FMC [26], fracture energy is defined as the amount of energy necessary to create one unit of area of crack and can be computed as:

$G_{F}=\frac{W_{F}}{A_{\text {lig }}}=\frac{W_{F}}{B\left(D-a_{0}\right)}=G_{f}$

where $W_{F}$ is the work of fracture (considered as the whole area under the load-displacement curve), $B$ is the sample thickness and $D-a_{0}$ is the ligament length.

As shown in Table 3 and according to Kim and Abu Al-Rub [27], the tensile strength and the elastic modulus (Fig. 6) tend to linearly increase with the addition of aggregate classes and the corresponding increase in the volume fraction of aggregates. The same trend is observed with the fracture energy. Thus, as a function of the increase in the volume fraction of aggregates, the crack path becomes more tortuous and leads to a higher fracture energy [28,29]. In other words, the high classes of aggregates (large diameter) increases the tortuosity of the crack path at large length scales while the fine classes (small diameter) tends to increase the tortuosity of the crack path at small length scales. In fact, with the addition of aggregates classes from the finest one, both effects act in the same time and tend to increase the "overall" length of the crack path and consequently of the resulting macroscopic fracture energy.

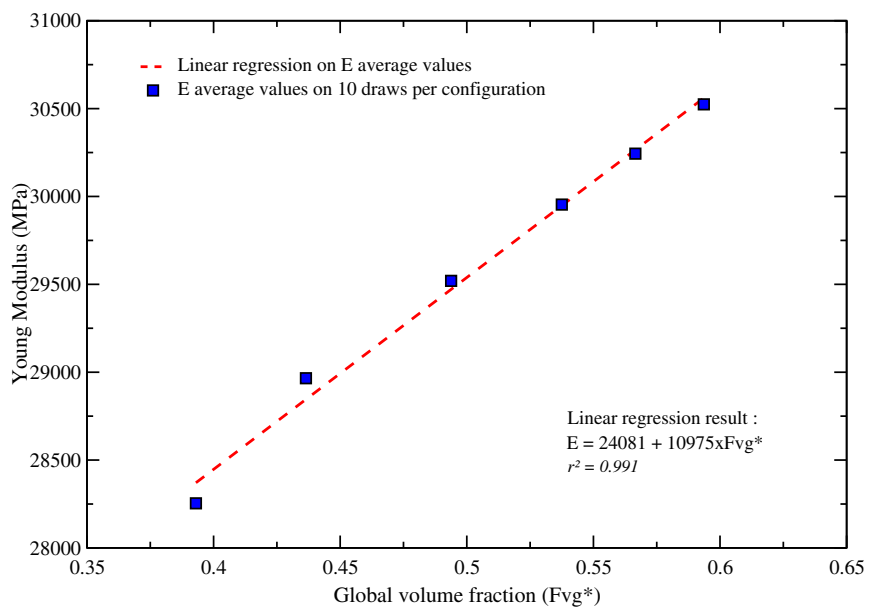

Fig. 6. Evolution of the Young modulus VS volume fraction of aggregates. 


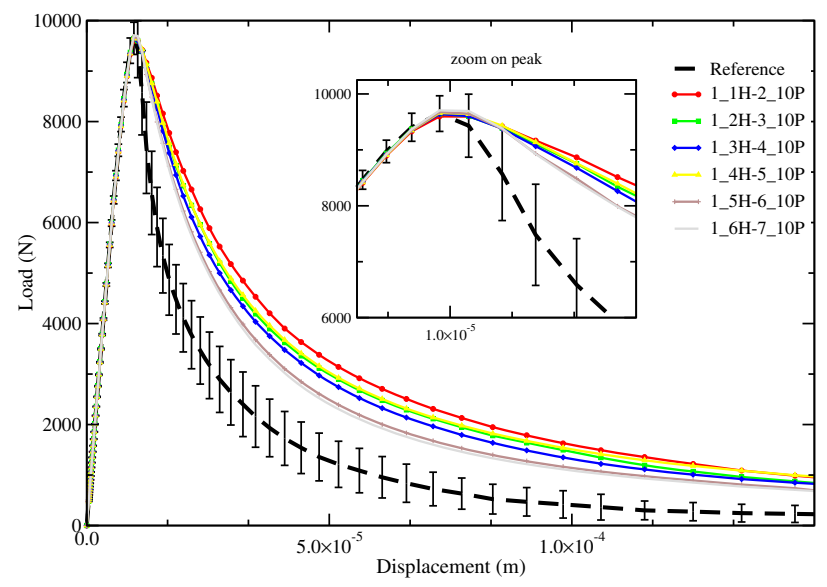

(a) with the use of $G_{\text {fmean }}$

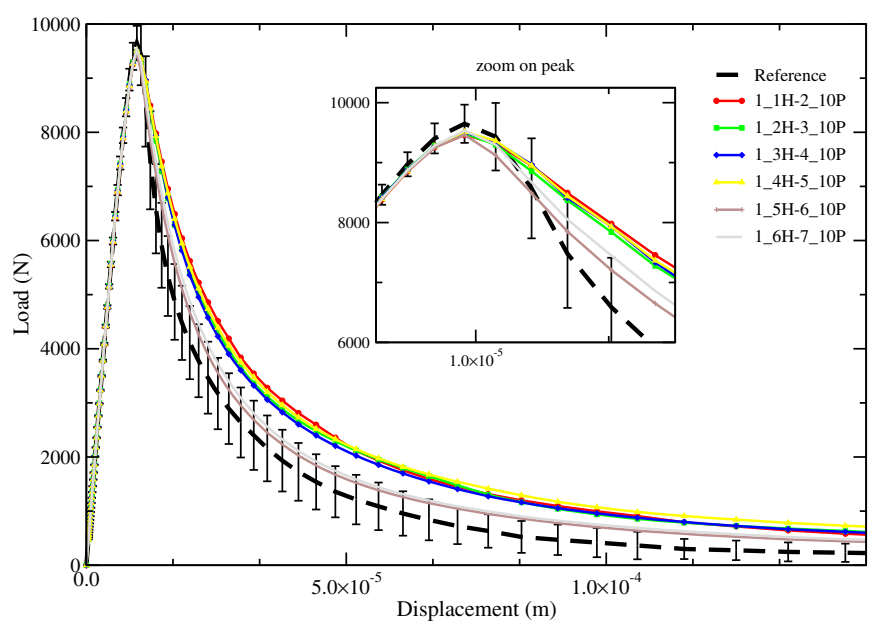

(b) with the use of $G_{f m i n}$

Fig. 7. Macroscopic response of simplified concretes (a) with the use of $G_{f m e a n}$ (b) with the use of $G_{f m i n}$.

Globally, standard deviations of elastic modulus and tensile strength are low. However, the standard deviation of the fracture energy is higher and increases as a function of the volume fraction of aggregates due to the scattering observed for the crack paths. For

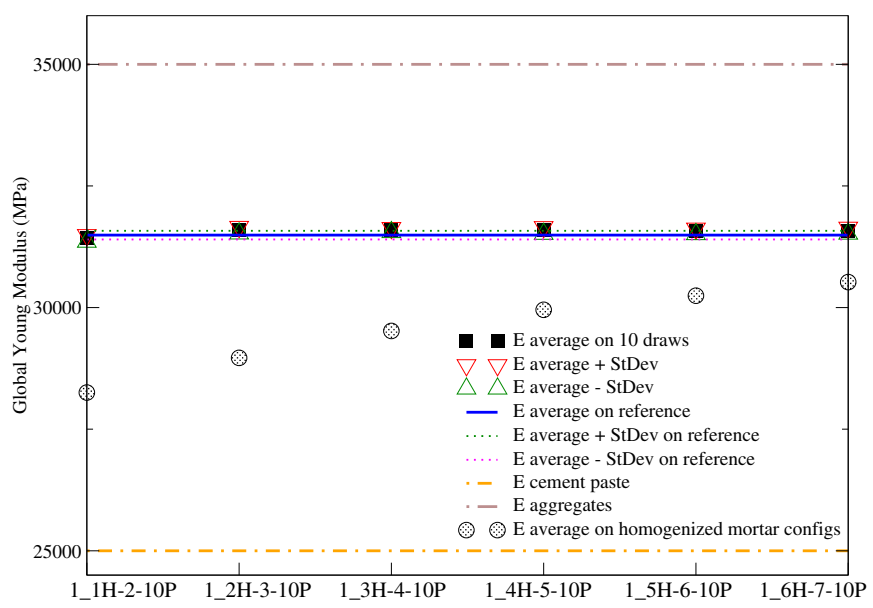

Fig. 8. Initial elastic modulus of the different simplified concretes. instance, a coefficient of variation of $25 \%$ can be noted for the configuration "1_6P-7_10M" corresponding to the highest volume fraction of aggregates (Fvg*). An Anderson-Darling Normality Test was performed and shows that the fracture energy values follow a normal distribution.

Thus, in order to take into account the scattered character of the fracture energy in the following parts of the study, three values of fracture energy are successively used: $G_{f m e a n}$ which is the average of 10 draws and $G_{f \min }$ and $G_{f \max }$ which are respectively the average value plus-minus 2 times the standard deviation (representation of $95 \%$ of the set for the normal distribution).

\subsection{Simulations of simplified concretes: Influence of the finer classes of aggregates}

\subsubsection{Comparison of mechanical responses}

Fig. 7 shows the load-displacement responses obtained from simplified concretes "1_(i)H-(i+1)_10P" compared to the response of the reference concrete. Fig. 7a corresponds to the responses obtained from a fracture energy of the homogenized mortar corresponding to $G_{\text {fmean }}$. It can be noted that the responses observed in the pre-peak regime (peak included) are analogous to the reference one, while in the post-peak regime, the responses overestimate that expected and consequently lead to overestimated values of the fracture energy

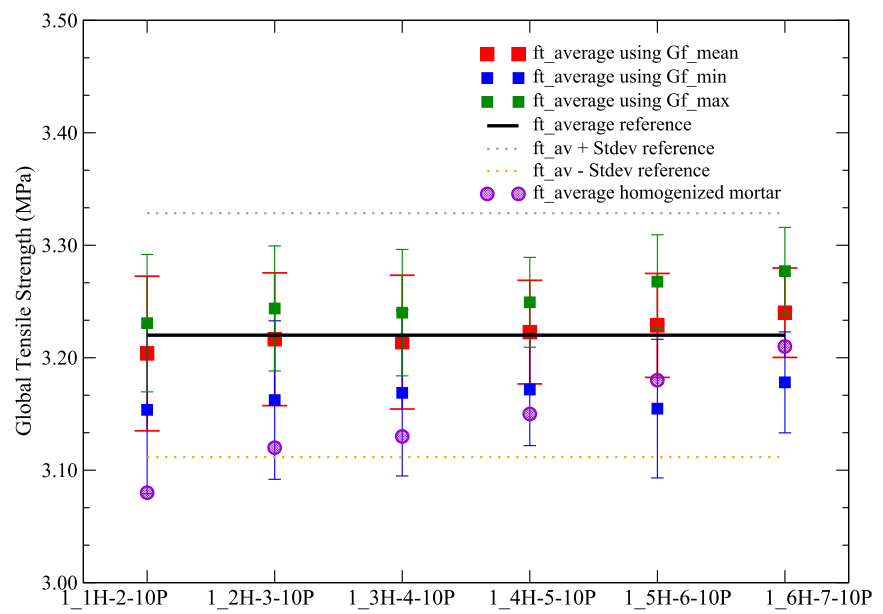

(a) global tensile strength

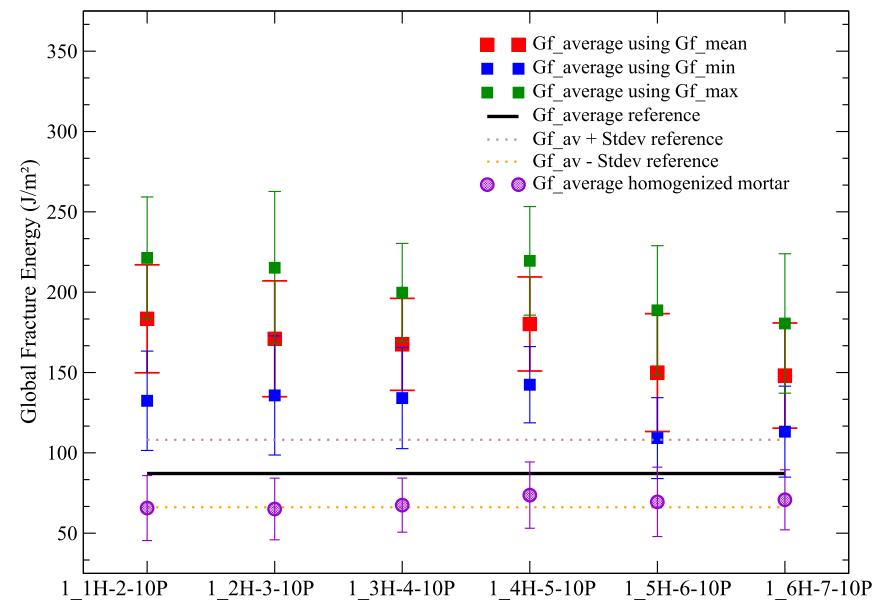

(b) global fracture energy

Fig. 9. Fracture properties of simplified concretes: (a) tensile strength $f_{t}$ and (b) fracture energy $G_{f}$ 

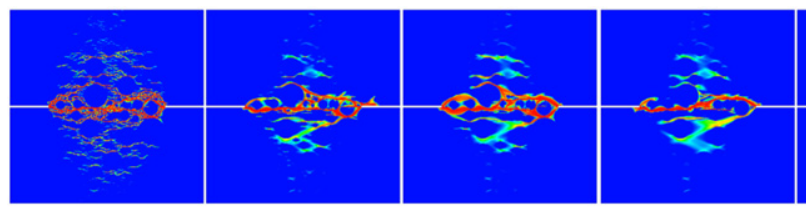

(a)
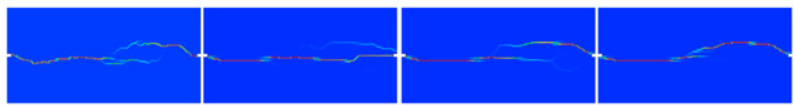

(b)
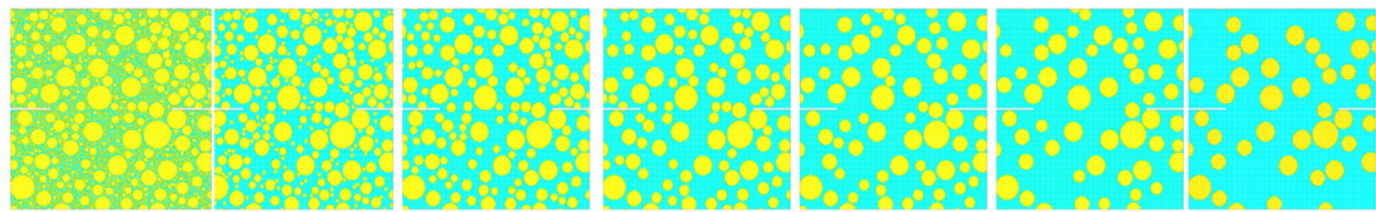

Reference

1_2H-3_10P

$1 \_3 \mathrm{H}-4 \_10 \mathrm{P}$

$1 \_4 \mathrm{H}-5 \_10 \mathrm{P}$

1_5H-6_10P 1_6H-7_10P

(c)

Fig. 10. Typical (a) damage fields and (b) crack path for the (c) reference and the simplified concrete.

compared to the reference one. In fact, damage to the simplified concretes localizes later compared to the reference simulation and leads to a residual stress which tends to increase the fracture energy. A surprising result is that the overestimation of the fracture energy is inversely proportional to the volume fraction of aggregates in the homogenized mortar.

The use of the lower values of the fracture energy $G_{f m i n}$ instead of the average one $G_{f m e a n}$ for the homogenized mortars reduces the overestimation of the fracture energy, as shown in Fig. 7b, but the same tendency can be observed in the effect of the volume fraction of aggregates in the homogenized mortars and a slight disagreement can be observed concerning the expected peak value.

\subsubsection{Comparison of elastic properties}

Fig. 8 shows the evolution of the elastic modulus of the simplified concretes. The values are quasi-constant and analogous to the reference with a maximum coefficient of variation of $0.55 \%$ (black square symbol), which seems to validate the homogenization method used to estimate the elastic properties of homogenized mortars.

\subsubsection{Comparison of fracture properties}

Fig. 9 shows the fracture properties $f_{t}$ and $G_{f}$ obtained for the 6 simplified concretes. The tensile strength $f_{t}$ values increase slowly throughout the homogenization process with a maximum average error compared to the reference one of about $0.6 \%$, which is smaller than the reference standard deviation even if the minimum and maximum values of $G_{f}$ are considered. Nevertheless, the best agreement for $f_{t}$ is obtained by using the average value of $G_{f}\left(G_{\text {fmean }}\right)$ for the homogenized mortar (HM). As expected from the load-displacement curves (Fig. 7) whose the magnitudes in the post-peak regime are greater than the one of the reference concrete, the total fracture energies of the simplified concrete overestimate the fracture energy corresponding to the reference one. This phenomenon is explained from Figs. 10,11 and 12 by damage fields more diffuse and continuous in simplified concretes compared to the one observed in the reference one and corresponding to an increase of the number of damage elements. Associated to an increase of the fracture energy of the corresponding homogenized mortars (Table 3), the increase of the number of damage elements lead to an overestimation of the total fracture energy. On the other, the total fracture energy $G_{f}$ of the simplified concretes tends to slightly decrease with the successive elimination of the finer classes of aggregates (Fig. 9) and this, in spite of the increase of the fracture energy of the corresponding homogenized mortars.

\subsubsection{Comparison of damage fields and crack paths}

The study of damage fields and resulting crack paths is illustrated here from one draw of the simplified concrete, i.e., considering a given granular skeleton where the smallest classes of aggregates are successively removed to the benefit of the homogenized mortar (Fig. 10). As shown in Fig. 10, the damage fields and the crack path (represented by deformation fields $\varepsilon_{y y}$ which seem to be sensitive to the real crack path) of the reference concrete exhibit pictures of the failure process which are analogous to those experimentally expected. Note that the damage fields and crack path plotted in Fig. 10 are considered as typical of the damage fields and crack paths observed on the 10 draws of granular skeleton. Removing aggregates leads to extended aggregate spacing and significantly impacts the dimensions of the damage zones. Indeed, one can observe that the

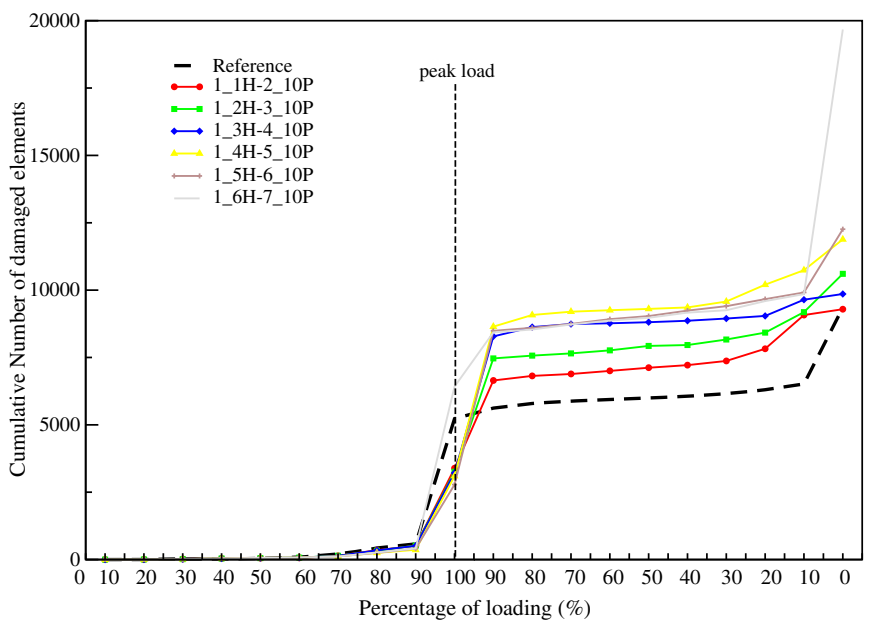

Fig. 11. Evolution of the number of damaged elements through the percentage of loading. 


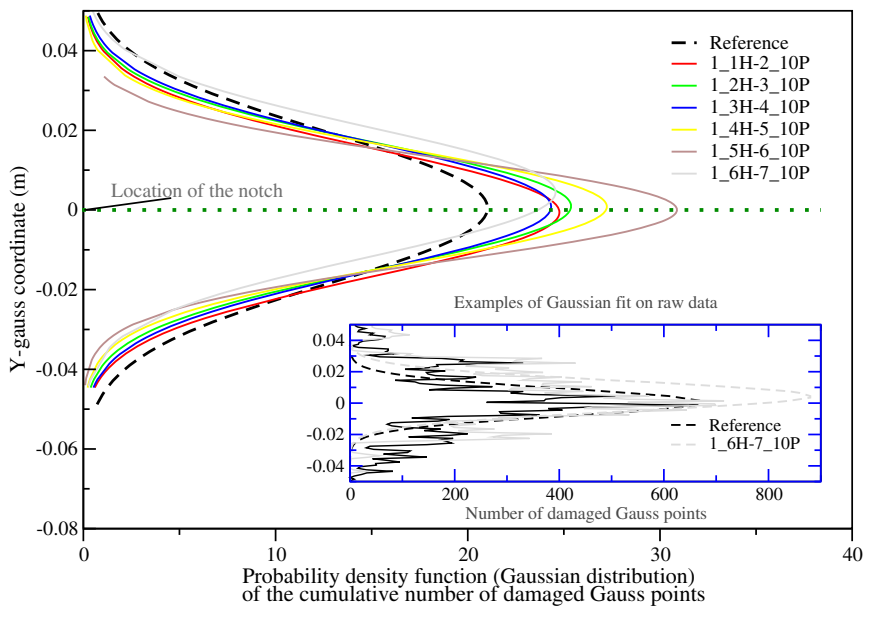

Fig. 12. Probability density function of the number of damaged Gauss points along $\mathrm{y}$-coordinate at the end of the simulations.

successive removal of lower aggregate classes induces a widening of the zone where FE exhibit greater damage than that observed in the reference simulation. This means that the damage field may not only be related to the aggregate sizes but also to the grain size contained in the cement matrix [30].

Therefore, the homogenization process of mortar leads to a loss of information at small length scales where the microstructure seems to play an important role. Moreover, the numerical results could be analyzed with the same Acoustic Emission (AE) analysis process [31], considering an analogy between recorded events from $\mathrm{AE}$ (corresponding to micro-crack nucleation) and numerical damaged elements [32]. It is on the basis of this latter remark that posttreatment results are achieved (Figs. 11, 12,13). Fig. 11 shows that removing aggregates from the granular skeleton increases the number of damage elements (i.e., elements for which $D \neq 0$ ) and this is true for the same number of FE constituting the mesh whatever the configuration studied. Since there are fewer aggregates, the localized damage zone is less confining. In addition,according the global response, the cumulative number of damage elements is clearly lesser in the reference concrete compared to the one of the reference mortar. On the other hand, Otsuka has shown that the development of the FPZ takes place between $30 \%$ in pre-peak regime and $70 \%$ in post-peak regime [33]. Fig. 11 is also in agreement with this latter result insofar as the number of damaged elements is highly varied in this loading interval. Another way to observe the different damage fields as a function of the considered configuration consists in estimating the probability density function of the cumulative number of damaged Gauss points along the y-coordinate (i.e., perpendicularly to the final crack path), as shown in Fig. 12 from the inserted graph exhibiting two examples of the obtained probability density function. Moreover, in order to compare the probability density functions, these ones can be fitted with a reasonable accuracy from a Gaussian distribution as shown in Fig. 12 (inserted graph). The resulting Gaussian distributions are plotted in Fig. 12 and exhibits a strong dependence of the maximum probability as a function of the configuration considered. Note that all the simplified concretes lead to maximum probabilities greater than those observed for the reference configuration and that this overestimation of the maximum probability increases as a function of the simplification of the granular skeleton of concrete. Moreover, if according to Haidar [34], the width of the FPZ might be estimated from a horizontal straight line which intercepts the probability density function curve at $20 \%$ of its maximum (related to the maximum number of events for a y-coordinate location), the FPZ width appears to be dependent on the granular

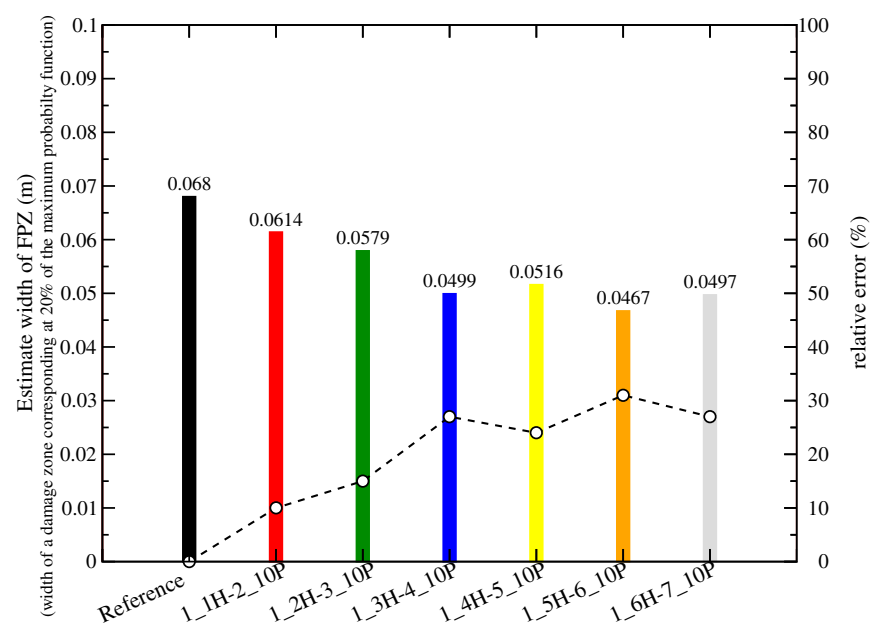

Fig. 13. Estimation of the width of the FPZ for the reference and simplified concretes (Haidar's method).

skeleton simplification and decreases with the simplification level (Fig. 13). Note that even if removing lower classes of aggregates tends to enlarge the damage fields, the probability of getting a damage element (or Gauss point) near the ligament is higher, and the concrete is more lacking in low aggregate classes. Thus, an error in the estimation of the width of the FPZ occurs with the simplification level.

\section{Conclusions}

The possibility of removing the smallest aggregate classes in the mesoscopic modeling of concrete due to time considerations was studied in this paper. To this end, a simulation procedure was established where lower aggregate classes were progressively removed from the granular skeleton to the benefit of a homogenized continuous mortar matrix. An isotropic damage model was used at the mesoscopic scale through a two-dimensional analysis which is useful for predicting the behavior qualitatively. This study was made for a parameter set that the cement paste is considering as the leak component, thus a standard concrete was studied. This model gives a good description of the failure process in concrete, ranging from diffuse damage to localization, towards a final crack. In addition, it is able to highlight the existence of branching cracks, due to (i) the mesostructure, (ii) the boundary conditions and (iii) the geometry of the specimen (notch). The impact of removing the lower classes of aggregates (small diameter) is analyzed by comparing the macroscopic behavior and the damage fields of the simplified concrete with different levels of granular skeleton simplification. The results show that the homogenization technique has no impact on the pre-peak region of the load-displacement curves, giving Young modulus and tensile strengths in agreement with those expected from the reference simulation. In other words, the homogenization procedure proposed in the paper is successfully applied for the elastic properties and it is clearly observed that the pre-peak load-displacement response is mainly governed by the elastic properties. However, the softening behaviors observed in the post-peak regime lead to an overestimation of the fracture energy. This is due to different damage fields and crack paths generated by the presence of small aggregates during damage localization and the softening process: small aggregates like sand are helpful for the strain and damage localization, and large aggregates bring tortuosity increasing dissipated energy for the failure. To conclude, the response obtained by the homogenization of one or several classes of aggregates can lead to an error 
in interpreting the global response and the damage fields, since different mechanisms induced by deleted aggregates are lost during the homogenization. Thus, for an accurate description of concrete failure, the whole granular skeleton must be considered even if this involves expensive computation time. The whole study is based on the consideration of a plain concrete, i.e., intergranular fracture. A new study might to be realize with the consideration of transgranular fracture (HPC) and/or a mixed inter-and trans-granular (thanks to the use of a Gaussian properties distributions for each component).

\section{Acknowledgments}

The MCIA (Mésoscentre de Calcul Intensif Aquitain), where all simulations on Cast $3 \mathrm{~m}^{\odot}$ were submitted, is gratefully acknowledged.

\section{References}

[1] I. Skarżyński, J. Tejchman, Calculations of fracture process zones on meso-scale in notched concrete beams subjected to three-point bending, Eur. J. Mech. A/Solids 29 (4) (2010) 746-760.

[2] Z. Wu, H. Rong, J. Zheng, F. Xu, W. Dong, An experimental investigation on the FPZ properties in concrete using digital image correlation technique, Eng. Fract. Mech. 78 (17) (2011) 2978-2990.

[3] J. Torrenti, Comportement mécanique du béton: bilan de six années de recherche, Études et recherches des LPC, Laboratoire central des ponts et chaussées. 1996.

[4] Y.B. Zaitsev, F.H. Wittmann, Simulation of crack propagation and failure of concrete, Matériaux et Constr. 14 (5) (1981) 357-365.

[5] J. Leite, V. Slowik, H. Mihashi, Computer simulation of fracture processes of concrete using mesolevel models of lattice structures, Cem. Concr. Res. 34 (6) (2004) 1025-1033.

[6] H. Mihashi, Material structure and tension softening properties of concrete, in: Z. Bažant (Ed.), Fracture Mechanics of Concrete Structure, Colorado, USA, 1992, pp. 239-250.

[7] G.V. Guinea, K. El-Sayed, C.G. Rocco, M. Elices, J. Planas, The effect of the bond between the matrix and the aggregates on the cracking mechanism and fracture parameters of concrete, Cem. Concr. Res. 32 (12) (2002) 1961-1970.

[8] M. Tasdemir, B. Karihaloo, Effect of type and volume fraction of aggregate on the fracture properties of concrete, Fracture Mechanics of Concrete Structure, Balkema, Cachan, France, 2001, pp. 123-129.

[9] A. Kwan, Z. Wang, H. Chan, Mesoscopic study of concrete II: nonlinear finite element analysis, Comput. Struct. 70 (5) (1999) 545-556.

[10] F. Camborde, C. Mariotti, F. Donzé, Numerical study of rock and concrete behaviour by discrete element modelling, Comput. Geotech. 27 (4) (2000) 225-247.

[11] P. Grassl, M. Jirásek, Meso-scale approach to modelling the fracture process zone of concrete subjected to uniaxial tension, Int. J. Solids Struct. 47 (7-8) (2010) 957-968.

[12] S. Fichant, C. La Borderie, G. Pijaudier-Cabot, Isotropic and anisotropic descriptions of damage in concrete structures, Mech. Cohesive-Frictional Mater. 4 (1999) 339-359.

[13] S. Fichant, Endommagement et anisotropie induite du béton de structures : modélisations approchées (Ph.D. thesis), Cachan, Ecole normale supérieure, 1996.
[14] J. Mazars, A description of micro- and macroscale damage of concrete structures, Eng. Fract. Mech. 25 (5-6) (1986) 729-737.

[15] Z. Bažant, B. Oh, Crack band theory for fracture of concrete, Mater. Struct. 16 (1983) 155-177.

[16] M. Matallah, C. La Borderie, O. Maurel, A practical method to estimate crack openings in concrete structures, Int. J. Numer. Anal. Methods Geomech. 34 (15) (2010) 1615-1633.

[17] C. La Borderie, C. Lawrence, T.D. N'Guyen, G. Nahas, A mesoscopic approach for a better understanding of the transition from diffuse damage to localized damage, Fracture Mechanics on Concrete and Concrete Structures, FraMCoS'7, 2010 ,

[18] T. N'Guyen, Apport de la modélisation mésoscopique dans la prédiction des écoulements dans les ouvrages en béton fissuré en conditions d'accident grave (Ph.D. thesis), Pau, 2010.

[19] R.A. Schmidt, Fracture-toughness testing of limestone, Exp. Mech. 16 (5) (1976) $161-167$.

[20] C.G. Broyden, A class of methods for solving nonlinear simultaneous equations, Math. Comput. 19 (92) (1965) 577-593.

[21] Z. Wang, A. Kwan, H. Chan, Mesoscopic study of concrete I: generation of random aggregate structure and finite element mesh, Comput. Struct. 70 (5) (1999) 533-544.

[22] I. Hager, Comportement à haute température des bétons à haute performance - évolution des principales propriétés mécaniques (Ph.D. thesis), Ecole Polytechnique de Cracovie, 2004.

[23] Z. Bažant, M. Tabbara, M. Kazemi, G. Pijaudier-Cabot, Random particle model for fracture of aggregate of fiber composites, J. Eng. Mech. 116 (8) (1990) 1686-1705.

[24] F. Grondin, H. Dumontet, A.B. Hamida, H. Boussa, Micromechanical contributions to the behaviour of cement-based materials: Two-scale modelling of cement paste and concrete in tension at high temperatures, Cem. Concr. Compos. 33 (3) (2011) 424-435.

[25] F. Grondin, G. Mounajed, A.B. Hamida, H. Dumontet, Digital concrete : a multi-scale approach for the concrete behavior, 2003,

[26] R. 50-FMC, Determination of the fracture energy of mortar and concrete by means of three-point bend tests on notched beams, Mater. Struct. 18 (106) (1985) 45-48.

[27] S. Kim, R. Abu Al-Rub, Meso-scale computational modeling of the plasticdamage response of cementitious composites, Cem. Concr. Res. 41 (3) (2011) 339-358.

[28] B.L. Karihaloo, Fracture Mechanics and Structural Concrete, Longman Scientific \& Technical. 1995.

[29] P. Petersson, Crack growth and development of fracture zones in plain concrete and similar materials (Ph.D. thesis), Division, Inst., 1981.

[30] J. Tejchman, J. Bobiński, Mesoscopic modelling of strain localization in plain concrete, Continuous and Discontinuous Modelling of Fracture in Concrete Using FEM, Springer Series in Geomechanics and Geoengineering Springer Berlin Heidelberg. 2013, pp. 343-405.

[31] J. Saliba, M. Matallah, A. Loukili, J.-P. Regoin, D. Grégoire, L. Verdon, G. PijaudierCabot, Experimental and numerical analysis of crack evolution in concrete through acoustic emission technique and mesoscale modelling, Eng. Fract. Mech. (2016)

[32] D. Grégoire, L. Rojas-Solano, V. Lefort, P. Grassl, G. Pijaudier-Cabot, Size and boundary effects during failure in quasi-brittle materials: Experimental and numerical investigations, Procedia Mater. Sci. 3 (2014) 1269-1278.

[33] K. Otsuka, H. Date, Fracture process zone in concrete tension specimen, Eng. Fract. Mech. 65 (2-3) (2000) 111-131.

[34] K. Haidar, Correlations between the internal length, the fracture process zone and size effect in mortar and model materials, Mater. Struct. 38 (276) (2005) 201-210. 